\title{
Enhancement of Signal-to-Noise Ratio and Phase Locking for Small Inputs by a Low-Threshold Outward Current in Auditory Neurons
}

\author{
Gytis Svirskis,, ${ }^{1,2}$ Vibhakar Kotak, ${ }^{1}$ Dan H. Sanes, ${ }^{1}$ and John Rinzel ${ }^{1,3}$ \\ ${ }^{1}$ Center for Neural Science, New York University, New York, New York 10003, 2Laboratory of Neurophysiology, Biomedical \\ Research Institute, Kaunas Medical University, 3000 Kaunas, Lithuania, and ${ }^{3}$ Courant Institute of Mathematical Sciences, \\ New York University, New York, New York 10012
}

\begin{abstract}
Neurons possess multiple voltage-dependent conductances specific for their function. To investigate how low-threshold outward currents improve the detection of small signals in a noisy background, we recorded from gerbil medial superior olivary (MSO) neurons in vitro. MSO neurons responded phasically, with a single spike to a step current injection. When bathed in dendrotoxin (DTX), most cells switched to tonic firing, suggesting that low-threshold potassium currents $\left(I_{\mathrm{KLT}}\right)$ participated in shaping these phasic responses. Neurons were stimulated with a computer-generated steady barrage of random inputs, mimicking weak synaptic conductance transients (the "noise"), together with a larger but still subthreshold postsynaptic conductance, EPSG (the "signal"). DTX reduced the signal-to-noise ratio (SNR), defined as the ratio of probability to fire in response to the EPSG and the probability to fire spontaneously in response to noise. The reduction was mainly attrib-
\end{abstract}

utable to the increase of spontaneous firing in DTX. The spiketriggered reverse correlation indicated that, for spike generation, the neuron with $I_{\mathrm{KLT}}$ required faster inward current transients. This narrow temporal integration window contributed to superior phase locking of firing to periodic stimuli before application of DTX. A computer model including HodgkinHuxley type conductances for spike generation and for $I_{\text {KLT }}$ (Rathouz and Trussell, 1998) showed similar response statistics. The dynamic low-threshold outward current increased SNR and the temporal precision of integration of weak subthreshold signals in auditory neurons by suppressing false positives.

Key words: medial superior olive; signal-to-noise ratio; phase locking; computer model; potassium conductance; slice recordings
Because of the temporal cues present in sound signals, the auditory nervous system provides a good opportunity to explore how multiple membrane currents influence signal integration (Trussell, 1999). The preservation of precise temporal information is crucial for coding and decoding, especially below $\sim 2 \mathrm{kHz}$ in mammals. Correspondingly, brainstem auditory neurons have very fast decaying synaptic currents (Raman and Trussell, 1992; Gardner et al., 1999) and fast low-threshold potassium currents $\left(I_{\mathrm{KLT}}\right)$ (Manis and Marx, 1991; Reyes et al., 1994; Rathouz and Trussell, 1998). They typically fire phasically, not tonically, for step current stimuli, presumably because of $I_{\mathrm{KLT}}$ (Manis and Marx, 1991; Reyes et al., 1994; Smith, 1995). Experimental and modeling studies suggest that such biophysical properties enhance the ability of auditory neurons to synchronize (phase lock) to strong sinusoidal stimuli (Oertel, 1983; Reyes et al., 1996; Rothman and Young, 1996) and, thus, to transmit more precisely the temporal information of the signal. However, it remains unknown how $I_{\text {KLT }}$ influences the integration of weak subthreshold inputs.

A common feature of afferent input to brainstem auditory nuclei is a high level of spontaneous activity. In the absence of a

Received April 30, 2002; revised Sept. 27, 2002; accepted Oct. 4, 2002.

This work was supported by National Institutes of Health/National Institute of Mental Health Grant MH62595-01 and National Science Foundation Grant DMS 0078420 (J.R. and G.S.) and National Institutes of Health Grant DC00540 (D.H.S.).

Correspondence should be addressed to John Rinzel, Center for Neural Science, 4 Washington Place, Room 809, New York, NY 10003-6621. E-mail: rinzel@cns.nyu.edu.

Copyright $\odot 2002$ Society for Neuroscience $\quad 0270-6474 / 02 / 2211019-07 \$ 15.00 / 0$ sound stimulus, this random firing can reach rates of $100 \mathrm{~Hz}$ (Liberman, 1978, 1982). Fibers with different spontaneous rates have different projections in the cochlear nucleus (Liberman, 1991, 1993), suggesting a specific role of the spontaneous random activity. It is well known that noise can facilitate the detection of weak signals in diverse natural and artificial systems (Bezrukov and Vodyanoy, 1995; Wiesenfeld and Moss, 1995). It is possible that neuronal spontaneous activity could serve a similar function, because it increases in lower auditory brainstem centers during performance (Ryan et al., 1984).

To explore the influence of $I_{\mathrm{KLT}}$ on the integration of subthreshold signals in the presence of noise, we recorded from gerbil medial superior olivary (MSO) neurons in vitro. MSO neurons and their avian analogs are implicated as coincidence detectors for sound localization (Jeffress, 1948; Goldberg and Brown, 1969; Carr and Konishi, 1990) and provide an opportunity to relate membrane properties with neuronal function. Notably, some MSO cells are spontaneously active in vivo, reflecting a significant amount of random spontaneous input (Goldberg and Brown, 1969; Young and Rubel, 1986; Carr and Konishi, 1990).

We stimulated MSO neurons with computer-generated currents that mimic transient random weak synaptic conductances. Because random neuronal activity is ubiquitous in the brain, stochastic inputs have been used extensively to investigate how biophysical features influence neuronal integrative and temporal processing characteristics (Bryant and Segundo, 1976; Softky and Koch, 1993; Mainen and Sejnowski, 1995; Hunter et al., 1998; Gauck and Jaeger, 2000). 
We found that blocking $I_{\mathrm{KLT}}$ in MSO neurons decreased the signal-to-noise ratio (SNR) when a subthreshold postsynaptic conductance (PSG) "signal" was injected together with weak random excitatory (EPSGs) and inhibitory (IPSGs) PSGs. Also, for weak random inputs with periodically modulated rate, $I_{\mathrm{KLT}}$ ensured better response synchronization (phase locking) with the stimulus. Computer simulations, which incorporate an experimentally determined $I_{\mathrm{KLT}}$ (Rathouz and Trussell, 1998), demonstrated similar benefits from $I_{\mathrm{KLT}}$ for detecting weak signals, increased SNR, and improved phase locking.

\section{MATERIALS AND METHODS}

Experimental. Gerbils (Meriones unguiculatus) aged postnatal day 8 (P8) to P17 were used to make $200-300 \mu \mathrm{m}$ coronal brain slices through the MSO. The artificial CSF (ACSF) contained the following (in mM): 125 $\mathrm{NaCl}, 4 \mathrm{KCl}, 1.2 \mathrm{KH}_{2} \mathrm{PO}_{4}, 1.3 \mathrm{MgSO}_{4}, 26 \mathrm{NaHCO}_{3}, 15$ glucose, 2.4 $\mathrm{CaCl}_{2}$, and $0.4 \mathrm{~L}$-ascorbic acid ( $\mathrm{pH} 7.3$ when bubbled with $95 \% \mathrm{O}_{2}-5 \%$ $\mathrm{CO}_{2}$ ). The ACSF was continuously superfused in the recording chamber at $4-5 \mathrm{ml} / \mathrm{min}$ at room temperature $\left(22-24^{\circ} \mathrm{C}\right)$. Whole-cell current-clamp recordings were obtained from MSO neurons (Axoclamp2A; Axon Instruments, Foster City, CA). The neurons were visually identified using infrared video microscopy (Olympus Optical, Tokyo, Japan). The internal patch solution contained the following (in $\mathrm{mM}$ ): 127.5 potassium gluconate, 0.6 EGTA, $10 \mathrm{HEPES}, 2 \mathrm{MgCl}_{2}, 5 \mathrm{KCl}, 2 \mathrm{ATP}$, and $0.3 \mathrm{GTP}$, $\mathrm{pH}$ 7.2. As described in Results, the following drugs were added to the ACSF: $10 \mathrm{~nm}$ dendrotoxin I (DTX) (Alomone Labs, Jerusalem, Israel), $20 \mathrm{~nm}$ dendrotoxin K (DTXK) (Alomone Labs), 4 mm kynurenic acid (Sigma, St. Louis, MO), and $1 \mu \mathrm{M}$ strychnine (Sigma). Recording electrodes were fabricated from borosilicate glass microcapillaries $(1.5 \mathrm{~mm}$ outer diameter), and their resistance ranged from 7 to $12 \mathrm{M} \Omega$.

Data acquisition and stimulus generation was performed under computer control at $10 \mathrm{kHz}$ using the programming package LabView (National Instruments, Austin, TX). The LabView program interfaced a module written in the $\mathrm{C}++$ programming language for computing stimulus and response statistics. We used the dynamic-clamp method for stimulus generation (Sharp et al., 1993; Reyes et al., 1996). Thus, the program determined in real time the time-varying current that was injected into the neuron according to the calculated conductance value and the measured instantaneous membrane potential. The program also saved spike times, defined as the time points when the membrane potential crossed $-5 \mathrm{mV}$ from below. The time course of injected current, for $20 \mathrm{msec}$, was kept in the computer memory buffer and used to calculate the spike-triggered reverse correlation (Bryant and Segundo, 1976; Mainen and Sejnowski, 1995).

To study the integration of weak transient signals in the presence of noise, a single EPSG was generated as a simple exponential decay from a step onset and time constant of $1 \mathrm{msec}$. This EPSG was to represent the coincident arrival of a few much smaller unitary-like EPSGs in the case of a weak auditory signal. This signal was repeated every $20 \mathrm{msec}$ while two trains of random smaller (on average) transient EPSGs and IPSGs (1 msec decay time constants) were delivered continuously representing the "noise." Each of the excitatory and inhibitory trains had exponentially distributed interspike intervals (independent Poisson trains) and the mean rate of $2 \mathrm{kHz}$. For random excitation, the reversal potential was 0 $\mathrm{mV}$, and, for inhibition, it was $-70 \mathrm{mV}$. The amplitude of synaptic conductance had an exponential distribution with a mean from 3 to $5 \mathrm{nS}$ for excitation and inhibition. The resulting spontaneous firing rate was equal to several tens of Hertz, in agreement with in vivo studies in the MSO (Goldberg and Brown, 1969). The amplitude for the signal EPSG was chosen so that the resulting EPSP was $<70 \%$ of the threshold for spike generation and was typically twofold to fivefold larger than the mean of random EPSGs. The stimulation lasted $200 \mathrm{sec}$ to gather several thousand events.

We studied the precision of phase locking by using a stimulus that consisted of random PSGs with a periodically modulated delivery rate. The probability, $p$, for a PSG to occur in the time interval $d t$ was equal to $d t \cdot R \cdot(M \cdot(\sin (2 \pi[t-D] / T)-1)+1)$ if the value of the expression was $>0$ and $p=0$ otherwise. Here, $R$ was a maximal rate, $M$ was modulation depth, $T$ was period, and $D$ was delay. $R=5$ and $2 \mathrm{kHz}$ for excitation and inhibition, respectively, $M=2, D=1 \mathrm{msec}$ for inhibition, $T=2 \mathrm{msec}$, and $d t=0.1 \mathrm{msec}$. We used this idealized description of input to simplify calculations done in real time. Although complex phenomenological models have been published for auditory nerve re- sponses (Zhang et al., 2001), we are not aware of similar models for the output from cochlear nucleus neurons. The stimulus was applied for 25 msec with rest intervals of $175 \mathrm{msec}$. The PSGs had exponentially distributed random amplitudes that were chosen so that each $25 \mathrm{msec}$ presentation generated no more than two spikes. The stimulation lasted $200 \mathrm{sec}$. Several thousand events were collected for computing a poststimulus time histogram (PSTH), and vector strength (VS) (Goldberg and Brown, 1969) was calculated as follows: VS $=\left(\left\langle\cos \left(2 \pi t_{j} / T\right)\right\rangle^{2}+\right.$ $\left.\left\langle\sin \left(2 \pi t_{j} / T\right)\right\rangle^{2}\right)^{1 / 2}$, where $t_{j}$ is the time of the $j$ th spike, and \langle\rangle indicates average over $j$.

Computational. We formulated and used a single compartment (lumped neuron) model with Hodgkin-Huxley type $\mathrm{Na}^{+}$and $\mathrm{K}^{+}$conductances for spike generation and a low-threshold potassium conductance. The parameters for the spike-generating currents were taken as described previously (Lytton and Sejnowski, 1991), with the voltage dependence of gating kinetics shifted rightward along the voltage axis by $5 \mathrm{mV}$. For $I_{\mathrm{KLT}}$, parameters were the same as characterized in avian nucleus magnocellularis cells (Rathouz and Trussell, 1998), with voltage dependence shifted rightward by $15 \mathrm{mV}$. The currents were calculated by using the following general equation: $I=g \cdot A_{\text {cell }} \cdot m^{p} \cdot h^{q}(V-E)$, where $g$ is specific conductance, $A_{\text {cell }}$ is membrane area, $p$ and $q$ represent the numbers of gating subunits, $V$ is membrane potential, and $E$ is reversal potential for the current. Activation and inactivation gating variables, $m$ and $h$, respectively, were governed by equations of the following form: $d u / d t=\left(u_{\infty}-u\right) / \tau_{u}$. The "steady state" value for the gating variable, $u$, was $u_{\infty}=\alpha /(\alpha+\beta)$, and time constant $\tau_{u}=1 /(\alpha+\beta)$; both $u_{\infty}$ and $\tau_{u}$ were voltage dependent: $\alpha=A_{0} \exp \left(-0.0393 z \gamma\left(V_{0.5}-V\right)\right), \beta=$ $B_{0} \exp \left(0.0393 z(1-\gamma)\left(V_{0.5}-V\right)\right)$, where $z$ is effective gating charge. The fast-activating sodium current had a reversal potential $E=50 \mathrm{mV}$. Its activation was described by the following parameters: $p=3, z=3.3, \gamma=$ $0.7, A_{0}=4.2 \mathrm{msec}^{-1}, B_{0}=4.2 \mathrm{msec}^{-1}$, and $V_{0.5}=-29.5 \mathrm{mV}$. Its inactivation had the following parameters: $q=1, z=-3.0, \gamma=0.27$, $A_{0}=0.09 \mathrm{msec}^{-1}, B_{0}=0.09 \mathrm{msec}^{-1}$, and $V_{0.5}=-40 \mathrm{mV}$. The time constants for activation and inactivation had limits to their minimal values set to 0.05 and $0.25 \mathrm{msec}$, respectively, to avoid exponents from occasional overflowing. The delayed rectifier conductance had only an activation gating variable, described by the following parameters: $E=$ $-90 \mathrm{mV}, p=4, z=3, \gamma=0.8, A_{0}=0.3 \mathrm{msec}^{-1}, B_{0}=0.3 \mathrm{msec}^{-1}, V_{0.5}=$ $-30 \mathrm{mV}$, and the minimal time constant for activation of $1 \mathrm{msec}$. The conductance $I_{\mathrm{KLT}}$ was described by the following parameters: $E=-90$ $\mathrm{mV}, p=1, z=2.88, \gamma=0.39, A_{0}=0.2 \mathrm{msec}^{-1}, B_{0}=0.17 \mathrm{msec}^{-1}$, and $V_{0.5}=-45 \mathrm{mV}$. This current did not inactivate.

The specific conductances, $g$, were $0.1,0.01$, and $0.005 \mathrm{nS} / \mu \mathrm{m}^{2}$ for $\mathrm{Na}^{+}, \mathrm{K}^{+}$, and $I_{\mathrm{KLT}}$ conductances, respectively. The area of the compartment, $A_{\text {cell }}$, was $10^{4} \mu \mathrm{m}^{2}$; specific conductance for membrane leakage was $3.333 * 10^{-3} \mathrm{nS} / \mu \mathrm{m}^{2}$, and specific capacitance was $10^{-5} \mathrm{nF} / \mu \mathrm{m}^{2}$, with the resulting time constant $\tau_{m}$ of 3 msec. The rest potential of the model was approximately $-60 \mathrm{mV}$, and the spike threshold was $\sim 20 \mathrm{mV}$ above rest.

The stimuli for the computational neuron were the same as in experiments, except for the conductance amplitudes of the PSGs. For the signal EPSG, the amplitude, $A$, was $60 \mathrm{nS}$. For the random PSGs, the mean amplitude, $a$, was $12 \mathrm{nS}$. For the periodically modulated stimulus, the mean conductance amplitude of PSGs was $30 \mathrm{nS}$. The numerical integration was performed with a fixed step-size second-order CrankNicholson scheme (Press et al., 1992), using a time step of $50 \mu \mathrm{sec}$.

\section{RESULTS}

\section{Experimental}

We recorded from visually identified MSO neurons. In animals older than P11, only 8 of 51 neurons responded with tonic firing; in the other cells, a step stimulus would not evoke more than a single spike (Fig. 1 $A$ ). Such a phasic firing pattern and the outward rectification, which appears near the resting membrane potential, $V_{\text {rest }}$, suggests the presence of an $I_{\mathrm{KLT}}$ in these MSO cells (Fig. $1 A$, inset, $E$, inset). This fast and strong outward current prevented spike generation in response to slowly rising current ramps $(n=3)$, although faster ramps of the same amplitude could evoke single spikes (Fig. 1C). Such effects of a low-threshold outward current were studied, experimentally (Ferragamo and Oertel, 2002) and computationally (Cai et al., 2000) with models, for octopus cells of the cochlear nucleus. 
A

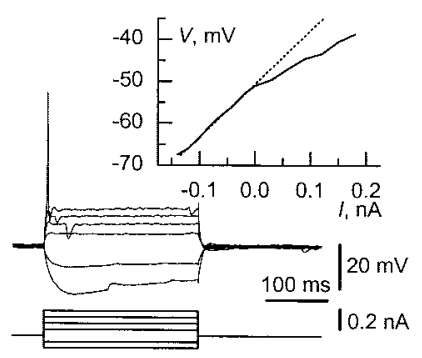

C

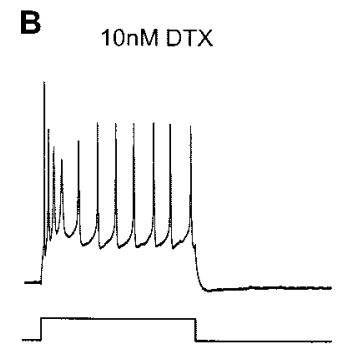

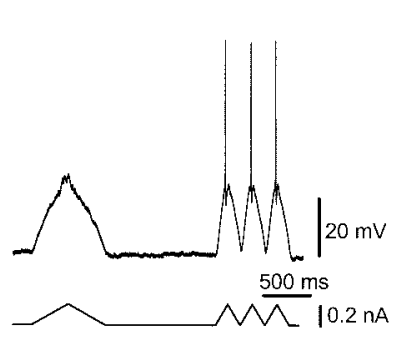

D

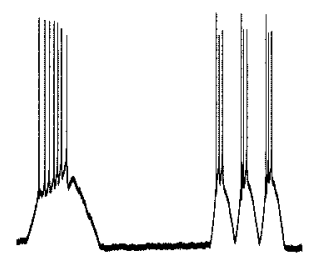

E

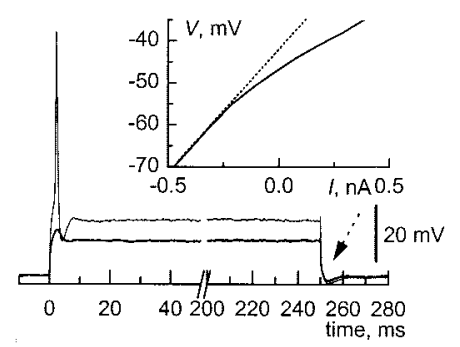

$\mathbf{F}$

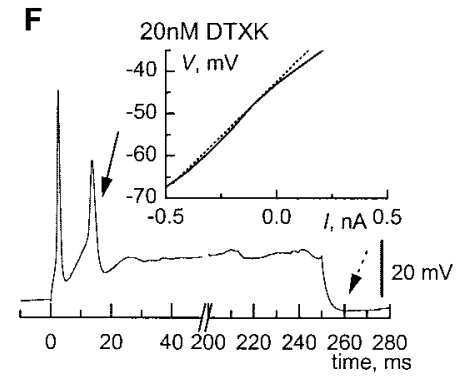

Figure 1. The firing properties of MSO neurons. $A$, In response to a step current injection, MSO neurons showed outward rectification and only a single spike when the stimulus exceeded the threshold (phasic firing). The steadystate current-voltage curve (inset) generated from the responses to step current stimuli showed that low-threshold outward rectification appeared near the resting membrane potential. Several spontaneous IPSPs were observed in these traces. The same properties suppressed firing in response to a slow triangular current-ramp stimulus, whereas faster stimuli evoked single spikes $(C)$. $B$, After an application of DTX, the cells fired tonically and responded with spikes to a slow current-ramp stimulus (D). E, In a more mature (P17) MSO neuron, outward current was activated at more hyperpolarized potentials, as can be seen in the current-voltage relationship (inset). F, DTXK did not induce tonic firing in the same neuron but lowered the spike threshold. The spike was evoked by the same current used for

the bottom trace in $E$. Note the afterdepolarization (arrow) after the spike and the absence of an undershoot (dotted arrows; compare $E, F$ ) after the step stimulus. These effects indicate that $I_{\mathrm{KLT}}$ was blocked by DTXK. The calibration in $B$ and $D$ are the same as in $A$ and $C$, respectively. $A$ and $B$ are from the same P14 neuron. $C$ and $D$ are from the same P12 neuron.

To confirm which membrane conductance is responsible for the outward rectification, we applied $10 \mathrm{~nm}$ DTX to the bathing solution. DTX is known to block some Kv1 family potassium channel subtypes (Hopkins et al., 1994; Robertson et al., 1996). The bathing solution also contained kynurenic acid (4 mM) and strychnine $(1 \mu \mathrm{M})$ to block the spontaneous synaptic activity that had increased in the presence of DTX. This solution had little influence on the resting membrane potential in slices at all ages tested (P12-P17): before application, the mean $V_{\text {rest }}$ was $-49.5 \pm$ $4.4 \mathrm{mV}$; afterward, it was $-50.6 \pm 4.9 \mathrm{mV}(n=12)$. The application of DTX-containing solution converted the phasic firing pattern of P12-P14 MSO neurons into tonic firing in 9 of 11 cases (Fig. 1B), and the cells could fire action potentials in response to slow ramps (Fig. $1 D$ ). In slices from older animals (P15-P17), application of DTX $(n=5)$ did not lead to tonic firing. Application of DTXK (P15-P18; $n=4)$, a specific blocker for Kv1.1 type of potassium channels (Wang et al., 1999), converted a phasic firing pattern to tonic in only one P15 cell, despite strong current injections that induced responses of $>30 \mathrm{mV}$ (see Discussion). However, in all cases, these blockers lowered the spike threshold (Fig. 1, compare $E, F$ ) and permitted an afterdepolarization after the first spike (Fig. $1 F$ ) that was presumably overridden by the subthreshold outward current in the control recordings.

To explore how the low-threshold outward current influenced the integration of small and random signals, we applied computer-generated dynamic-clamp stimuli. In particular, we considered how well the occurrence of a subthreshold signal EPSG was detected in a background of ongoing smaller random excitatory and inhibitory transients, the noise (Fig. 2A). For auditory neurons, this signal EPSG could represent the synchronized arrival of unitary inputs in the case of a very weak auditory stimulus. The signal EPSG could not evoke a spike in the absence of random input. However, as seen in the computed PSTH (see Materials and Methods), when the signal EPSG occurred together with noise, there was a sharp increase of firing probability over the spontaneous firing levels (Fig. 2B). To measure the efficiency of signal detection, we defined and computed the SNR as the ratio of the increased firing rate in response to the signal EPSG (deviation of PSTH value from its baseline) and the spontaneous firing rate in response to noise (Fig. $2 B$, inset). The application of DTX or DTXK, which lowered spike threshold (Fig. 1, compare $E, F$ ), increased the spontaneous rate significantly, thus reducing the SNR $(n=11)$. That is, in the control case, $I_{\mathrm{KLT}}$ prevents some of the temporally summated random inputs from generating spikes, whereas the larger amplitude (but subthreshold) and faster signal can ride on the noise and "break through" before the transient outward rectification is fully recruited.

To observe the transient net input current that develops before spike generation, we performed a reverse correlation analysis to compute the average spike-triggered dynamic-clamp "synaptic" current (Fig. 2C-E) (see Materials and Methods). As expected, it was predominantly an inward (depolarizing) current that developed on a time scale of 2-3 msec. The application of DTX or DTXK changed the time course of the mean spike-triggering input current: it developed more slowly, as can be seen for a single trace for one cell (Fig. 2C) and for the average of traces from seven neurons (Fig. 2D,E). The maximal rate of mean injected current calculated for a $0.5 \mathrm{msec}$ time window was significantly slower after block of $I_{\mathrm{KLT}}(n=7 ; p<0.05$; paired Student's $t$ test $)$ and decreased from $0.8 \pm 0.38$ to $0.5 \pm 0.3 \mathrm{nA} / \mathrm{msec}$. The faster dynamics of the spike-triggering current as mediated by $I_{\mathrm{KLT}}$ further implicated the role of $I_{\mathrm{KLT}}$ in enhancing the precision of temporal processing. To explore further the effect of this narrowed temporal window for integration, we applied weak random periodically modulated stimuli (see Materials and Methods). For such a weak stimulus, which evoked only one or two spikes per trial, the phase locking was improved (Fig. $2 F$ ), and the vector strength was, on average, 1.5 times greater in control recordings compared with those obtained in the presence of DTX $(n=5)$. 
Figure 2. The firing statistics of a P17 MSO neuron in response to weak and noisy stimuli. $A$, Traces of membrane potential illustrating random and signal evoked firing. $B$, The PSTH for the response to a subthreshold EPSP signal in the presence of smaller random excitatory and inhibitory input transients. The EPSG, generated by dynamic clamp, in addition to the random input caused a sharp increase in the probability to fire. DTX increased the spontaneous firing rate and thereby reduced the SNR several times (inset shows difference of PSTH probability from baseline and then divided by baseline). $C$, Spiketriggered reverse correlation exhibited a hyperpolarizing component followed by excitation in control conditions. In the presence of DTX, an average spikeevoking current developed slower. The error bars mark the SD for the injected current; the $x$ symbol denotes the mean current value. The average over a pop-

ulation of seven neurons of the mean injected current (over trials as in $C$ ) for before $(D)$ and after $(E)$ application of DTX or DTXK have similar properties as for a single neuron. The error bars mark the SD (shown only below the average) for each time point. The baselines were subtracted. $F$, The PSTH and period histogram (inset) showed phase-locked firing to a periodically modulated stimulus. After DTX, the vector strength of phase locking decreased by nearly one-half.

Figure 3. Statistical response properties of a model with Hodgkin-Huxley type conductances. $A$, The PSTH for a subthreshold EPSG in the presence of random input. The low-threshold outward current reduced the spontaneous firing rate to $2 \mathrm{~Hz}$ but increased the signal-tonoise ratio (inset). $B$, The spike-triggered reverse correlation had the same shape as for the recorded neurons, with a hyperpolarizing component and faster dynamics for the spike-evoking current in the model with $I_{\mathrm{KLT}}$. The traces were

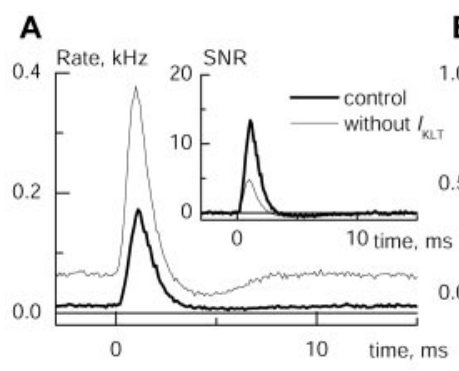

B

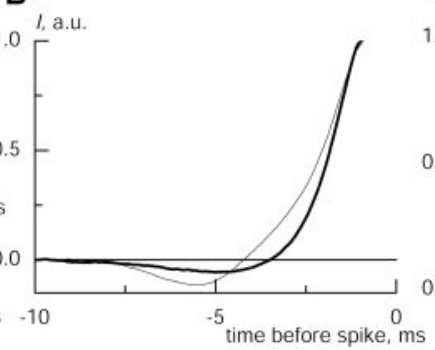

$\mathbf{E}$
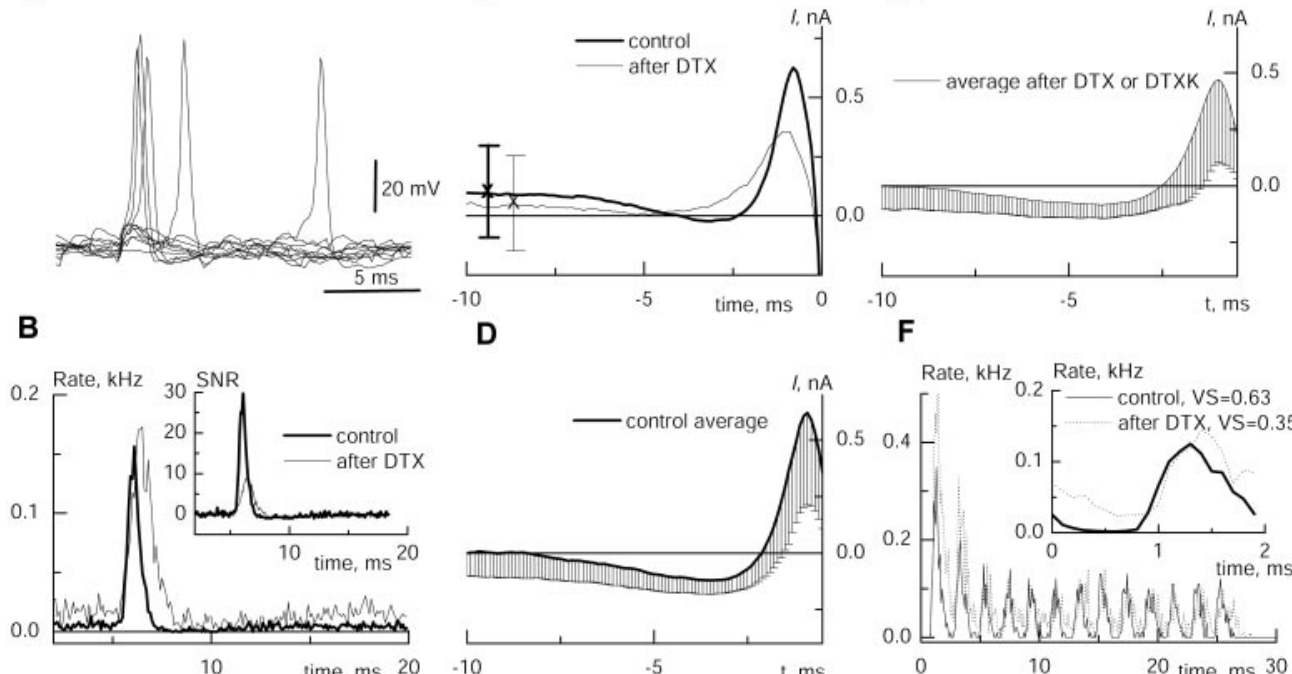

normalized, and baselines were subtracted to compare more easily the time course shapes. a.u., Arbitrary units. $C$, Vector strength (VS) versus frequency, for periodically modulated stimulus. The model with $I_{\mathrm{KLT}}$ phase locked more strongly at each frequency.

\section{Computational}

To confirm that the $I_{\mathrm{KLT}}$ could account for the observed changes in the integration of weak noisy signals, we performed a modeling study. The model has two voltage-dependent conductances for spike generation and a fast-activating, low-threshold outward conductance; the stimuli were the same as those used in the whole-cell recordings (see Materials and Methods). For implementing $I_{\mathrm{KLT}}$ in the model, we modified the parameter values as obtained from the avian nucleus magnocellularis (Rathouz and Trussell, 1998) by shifting the voltage threshold for activationdeactivation upward by $15 \mathrm{mV}$ to match better our observations in MSO. Because our goal for the computational work was to achieve semiquantitative comparison with our experimentally observed responses and to obtain insight by demonstrating qualitative parametric dependencies, we did not perform extensive parameter adjustments to get a detailed quantitative replication of experimental results. Nevertheless, our model shows all of the basic response properties of MSO neurons when presented with the same (but amplitude adjusted) stimuli as in our experiments (see Materials and Methods). It exhibits phasic firing and tonic firing when $I_{\mathrm{KLT}}$ was eliminated (data not shown). The presence of $I_{\mathrm{KLT}}$ in the model makes the spontaneous firing rate much lower than in the model without $I_{\mathrm{KLT}}$ (Fig. $3 A$ ). The presence of $I_{\mathrm{KLT}}$ reduces the response to the signal EPSG as well but not as much as for the spontaneous firing probability. Consequently, $I_{\mathrm{KLT}}$ increases the signal-to-noise ratio (Fig. $3 A$, inset). The mean input current that precedes spike generation has the same shape as in MSO neurons (Fig. 3B), and it has a faster mean time course with $I_{\mathrm{KLT}}$ compared with when $I_{\mathrm{KLT}}$ is eliminated. In addition, the mean spike-triggering current has a hyperpolarizing undershoot before rising to its maximum (see Discussion). In agreement with the experimental data, the phase locking is better (i.e., the vector strength is higher) in the model when $I_{\mathrm{KLT}}$ is present for all frequencies tested (Fig. 3C).

We applied the model to characterize how the influence of $I_{\mathrm{KLT}}$ on subthreshold integration depends on various parameters, including input amplitudes and the gating time scale of $I_{\mathrm{KLT}}$. First, we describe how signal detection depends on the signal and noise strengths. As a measure of detection, we use a probability ratio. Consider the interval in which the signal has its effect, $0 \leq$ $t \leq \Delta$, where $\Delta$ is $\sim 3 \mathrm{msec}$. The probability to fire spontaneously, if there were no signal, is $P_{\mathrm{N}} \Delta$ where $P_{\mathrm{N}}$ is the floor level of the PSTH. The probability $P_{\mathrm{S}}$ to fire with the signal present is the integral of the PSTH over $0 \leq t \leq \Delta$. Of interest to us is $P_{\mathrm{SN}}=$ $\left(P_{\mathrm{S}}-P_{\mathrm{N}}\right) / P_{\mathrm{N}}$, the ratio of the probabilities for a spike to be generated as a result of the signal or spontaneously.

$P_{\mathrm{SN}}$ decreases very strongly (Fig. $4 A$ ) as the mean amplitude, $a$, for the randomly occurring weak PSGs increases. The effect of 


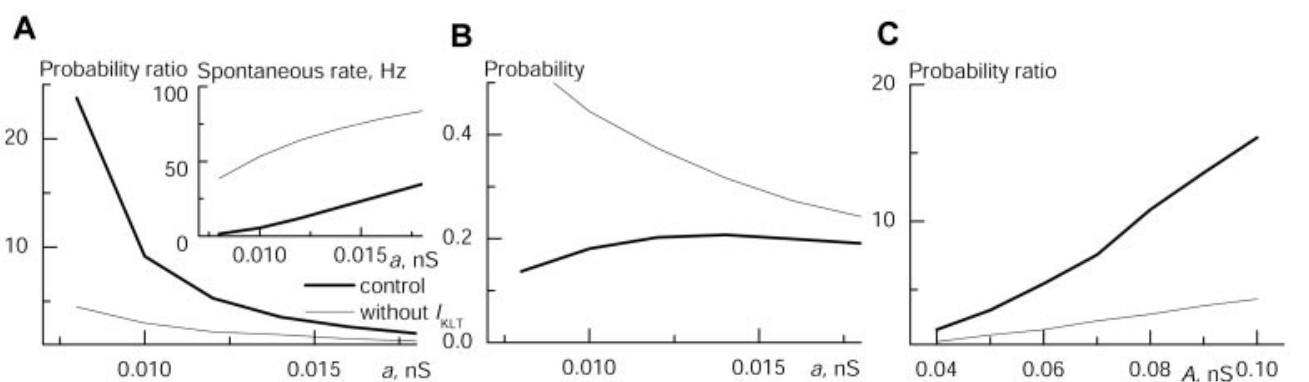

the signal grows. The $I_{\mathrm{KLT}}$ effect is strongest for the largest amplitude.

A

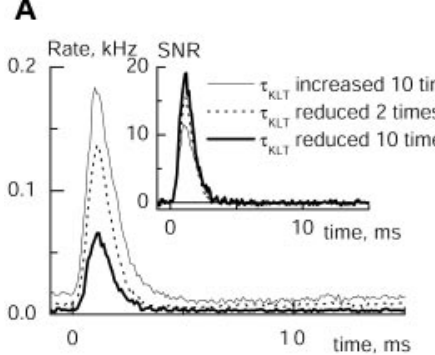

B

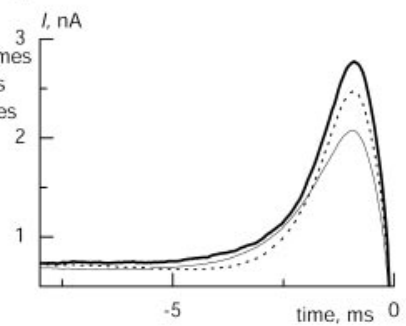

C

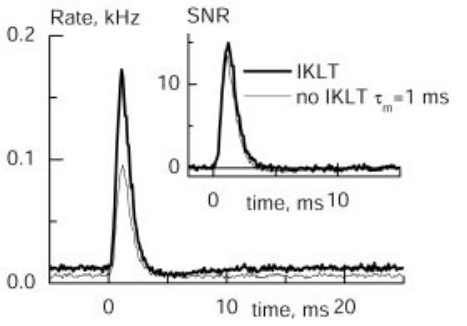

Figure 5. Influence of $I_{\mathrm{KLT}}$ properties on small-signal integration in the model. $A$, Very slow $I_{\mathrm{KLT}}$ activation increases spontaneous activity, whereas very fast activation suppresses strongly both the response to the signal and spontaneous activity. Parameters $A_{0}$ and $B_{0}$ were changed to speed up or slow down the activation of $I_{\mathrm{KLT}}$. $B$, Spike-triggered reverse correlation shows fastest current transients for the model with $I_{\mathrm{KLT}}$ having intermediate activation rate. $C$, When $I_{\mathrm{KLT}}$ is replaced by an increased leak conductance in the model, the SNR is little changed, although suppression of the response was stronger without $I_{\mathrm{KLT}}$.

$I_{\mathrm{KLT}}$ on increasing this ratio of spike-generating probabilities is largest for the weakest noise. This result can be explained by the strong increase of the spontaneous firing rate $P_{\mathrm{N}}$ with the mean synaptic amplitude, $a$ (Fig. $4 A$, inset).

Another comparison is obtained by considering the increased probability (beyond $P_{\mathrm{N}}$ ) for generating a spike that is contributed by the signal EPSG. This value, $P_{\mathrm{S}}-P_{\mathrm{N}}$, also depends on $a$ but non-monotonically (Fig. $4 B$; note that, for the case without $I_{\mathrm{KLT}}$, the peak occurs at a smaller $a$ value than is seen on this scale). This plot for the control case shows explicitly that a sufficient amount of noise is needed to detect the subthreshold signal; as $a$ decreases toward zero, so does $P_{\mathrm{S}}-P_{\mathrm{N}}$. For stronger noise, this probability is also reduced because the signal becomes buried in the noise; one factor is that the membrane potential can be randomly reduced at the time of the EPSG.

Of course, the probability to generate a spike also depends on the amplitude, $A$, of the signal EPSG. If $A$ is increased, the probability ratio $P_{\mathrm{SN}}$ likewise increases. In this case, the effect of $I_{\mathrm{KLT}}$ on the ratio of probabilities is largest for the strongest $A$ (Fig. 4C).

Although the integrative effects of $I_{\mathrm{KLT}}$ are often attributed to the increased subthreshold conductance (and, hence, reduced integration time) (Reyes et al., 1994; Oertel et al., 2000), little attention has been given to how the dynamic properties per se of $I_{\mathrm{KLT}}$ affect small-signal detection. We performed additional simulations to address this issue. First, if the activation of $I_{\mathrm{KLT}}$ is very slow, then little conductance would be activated over time attributable to fast membrane potential fluctuations (with mean below the threshold of $I_{\mathrm{KLT}}$ ). In agreement, when we slowed the gating kinetics of $I_{\mathrm{KLT}}$ by a factor of 10 in the model, the spontaneous firing rate increased and the signal-to-noise ratio decreased over the control values (Fig. $5 A$ ).
Figure 4. Influence of $I_{\mathrm{KLT}}$ on the integration of small signals with different amplitude and in the presence of noise with various strengths. $A$, The ratio of the probabilities to generate a spike attributable to the signal spontaneously decreases when the amplitude $a$ of the random EPSGs increases. Note that the increase of SNR by $I_{\mathrm{KLT}}$ is strongest for the weakest noise. $B$, The probability to generate a spike in response to the signal calculated as an integral of rate larger than spontaneous rate. Both curves have abilities increases when the amplitude of (a) 
Trussell, 1998). We recorded from gerbil MSO neurons and found that the low-threshold DTX-sensitive potassium current increased the signal-to-noise ratio for a subthreshold signal in the presence of weak random input. $I_{\mathrm{KLT}}$ also increased the ability of the neuron to spike in synchrony or phase lock with a periodically modulated barrage of weak synaptic inputs. A computational model with Hodgkin-Huxley type conductances for spike generation and $I_{\mathrm{KLT}}$ had the same qualitative integration properties for noisy signals as did MSO neurons in vitro. Both the voltage and time dependence of the subthreshold outward current contribute to enhancing weak signal integration. If the $I_{\mathrm{KLT}}$ of the model is replaced by an increased constant leakage conductance, then the SNR could be increased up to that of the model with $I_{\mathrm{KLT}}$, but the response to the signal is significantly reduced.

The ability of neuronal properties to influence signal integration in the presence of noise was extensively investigated previously. For example, such studies suggested that an interaction between noisy signals and voltage-dependent currents could bring about increased temporal precision (Gauck and Jaeger, 2000), improved coincidence detection (Softky and Koch, 1993; Softky, 1994), increased information transfer (Manwani and Koch, 1999), and spike timing reliability (Mainen and Sejnowski, 1995; Hunter et al., 1998). Our study concentrated on the effects of one particular current, $I_{\mathrm{KLT}}$, and tried to elucidate the mechanisms by which neurons could improve small-signal detection.

Although DTX decreased SNR and degraded phase locking in each neuron tested, for animals older than P15, DTX or DTXK in the majority of cases did not convert the firing mode from phasic to tonic. It was unlikely that the phasic property, remaining after these blockers, was caused by another low-threshold potassium current. Several changes that we observed in the neuronal response after application of DTX were consistent with elimination of a low-threshold non-inactivating outward current: the current-voltage input relationship was almost linearized (Fig. $1 F$, inset), an afterdepolarization appeared after the spike (Fig. $1 F$, arrow), and there was no undershoot after termination of a current step (Fig. $1 F$, dotted arrow). This remaining phasic behavior could possibly be attributable to a maturational change in the inactivation properties of sodium channels (Sada et al., 1995; Schmid and Guenther, 1998).

In a separate computational study (Svirskis and Rinzel, 2003), we showed that an integrate-and-fire type model without an $I_{\mathrm{KLT}}$ but with an idealized subthreshold inward current can behave phasically if the inward current inactivates with an appropriate time scale and in a voltage range below its activation threshold; the model also shows improved SNR compared with the case with no subthreshold inactivation. Thus, multiple forms of subthreshold fast, but not necessarily instantaneous, negative feedback can contribute to enhancing temporal processing. The failure of DTX to eliminate the phasic firing pattern demonstrates that $I_{\mathrm{KLT}}$ in MSO neurons contributes to subthreshold integration in ways other than just to generate phasic responsiveness.

We emphasized that the dynamic aspects (i.e., the time scale of the gating kinetics) of $I_{\mathrm{KLT}}$ activation are important for understanding how $I_{\mathrm{KLT}}$ affects signal throughput (Fig. 5A,B). Fast just-suprathreshold depolarizing inputs can squeeze through to spike threshold before $I_{\mathrm{KLT}}$ is activated, whereas slower ones that would be suprathreshold, if the membrane conductance remained at its resting level, will now be rendered subthreshold because there will be adequate time for reducing the membrane resistance by activating the conductance of $I_{\mathrm{KLT}}$. In this sense, $I_{\mathrm{KLT}}$ effectively acts to implement a dynamic threshold. Here we considered a spontaneous state of noisy fast weak inputs. A few nearly coincident ones provide fast depolarization that can lead to a spontaneous firing. Occasionally, some of these, not so nearly coincident, will summate temporally to create a slower transient that would be suprathreshold if it were not falling into the temporal window for recruiting $I_{\mathrm{KLT}}$. Therefore, $I_{\mathrm{KLT}}$ can eliminate false positives.

In nucleus magnocellularis neurons (Rathouz and Trussell, 1998), $I_{\mathrm{KLT}}$ is partially activated at rest. In many of the cells from which we recorded, the outward potassium current was apparently barely activated near $V_{\text {rest }}$ (Fig. $1 A$, inset, $E$, inset) because DTX did not change $V_{\text {rest }}$ significantly $(-1.1 \pm 2.6 \mathrm{mV})$. For this reason, in the model, we shifted the voltage dependence of $I_{\mathrm{KLT}}$ as shown previously (Rathouz and Trussell, 1998) to more depolarized potentials, so that the activated conductance is small at rest. Such a shift is consistent with observations in other mammalian auditory neurons that low-threshold potassium currents activate at more depolarized potentials (Brew and Forsythe, 1995; Bal and Oertel, 2001). For the same reason, we suppose that changes in the current time course obtained by spike-triggered reverse correlation before and after blocking $I_{\mathrm{KLT}}$ (Fig. $2 C-E$ ) are not attributable to the changes in the mean $V_{\text {rest }}$.

To demonstrate the significance of $I_{\mathrm{KLT}}$ on small-signal integration, we used random input together with a larger but still subthreshold EPSG as a distinguished signal. In auditory neurons, such a stimulus could represent the coincidence of synaptic EPSGs during very weak auditory input. During such a subthreshold transient auditory stimulus, the rate of firing on the auditory nerve may be little different from spontaneous. However, before reaching the MSO, these auditory nerve spike trains converge on and pass through the anteroventral cochlear nucleus, in which vector strength between the input and the output can increase (Joris et al., 1994; Rothman and Young, 1996).

We computed the spike-triggered reverse correlation for the input current to show that $I_{\mathrm{KLT}}$ shortens the temporal integration window. The mean current calculated from reverse correlation was used to illustrate temporal changes in the current preceding spike generation. To confirm that the mean current reflects the most probable values of the current transients, we calculated, for our computational model, the probability densities for each time $t$ preceding spike generation (Bryant and Segundo, 1976). For each $t$, the probability density function for the random input current had a single peak; it was not multimodal. Thus, the mean value should reflect the most probable value for the spiketriggering current.

We notice the hyperpolarizing undershoots (Fig. $2 C-E, 3 B$ ) in these average currents a few milliseconds preceding the sharp depolarizing rise, in both our theoretical and experimental results. It is tempting to attach an interpretation to these "dips;" for example, in Figure $2 C$, we expect that a removal of some $I_{\mathrm{KLT}}$ conductance by hyperpolarization followed by fast excitation could contribute to the generation of some spikes. However, multiple factors likely play a role (in Fig. $3 B$, there is a dip even without $I_{\text {KLT }}$ present) and dissecting these factors will be for our future work.

Various investigators have proposed that low-threshold potassium currents help to suppress weak subthreshold inputs but improve the temporal precision of integration of strong suprathreshold inputs (Oertel, 1983; Manis and Marx, 1991; Reyes et al., 1994; Brew and Forsythe, 1995; Rothman and Young, 1996; Rathouz and Trussell, 1998). We add another perspective by considering the integration of subthreshold inputs in the presence 
of weak noise and focusing on the signal-to-noise ratio. It is important to stress that, in the case of subthreshold inputs, the noise is necessary so that the neuron can detect a potentially meaningful subthreshold signal. On the other hand, if the signal is absent, noise creates false positives. The low-threshold potassium current in such circumstances plays two roles: it allows the noise to aid in signal detection, and it suppresses false positives if the signal is absent. It could be that such a scenario is also applicable to stronger auditory signals because in vivo spikes in MSO and nucleus laminaris are not evoked on every single cycle of stimulation (Goldberg and Brown, 1969; Carr and Konishi, 1990). Thus, we conclude that the low-threshold potassium current plays a major role in the processing not only of strong but also of weak auditory signals.

\section{REFERENCES}

Bal R, Oertel D (2001) Potassium currents in octopus cells of the mammalian cochlear nucleus. J Neurophysiol 86:2299-2311.

Bezrukov SM, Vodyanoy I (1995) Noise-induced enhancement of signal transduction across voltage-dependent ion channels. Nature 378:362-364.

Brew HM, Forsythe ID (1995) Two voltage-dependent $\mathrm{K}^{+}$conductances with complementary functions in postsynaptic integration at a central auditory synapse. J Neurosci 15:8011-8022.

Bryant HL, Segundo JP (1976) Spike initiation by transmembrane current: a white-noise analysis. J Physiol (Lond) 260:279-314.

Cai Y, McGee J, Walsh EJ (2000) Contributions of ion conductances to the onset responses of octopus cells in the ventral cochlear nucleus: simulation results. J Neurophysiol 83:301-314.

Carr CE, Konishi M (1990) A circuit for detection of interaural time differences in the brain stem of the barn owl. J Neurosci 10:3227-3246.

Ferragamo MJ, Oertel D (2002) Octopus cells of the mammalian ventral cochlear nucleus sense the rate of depolarization. J Neurophysiol 87:2262-2270.

Gardner SM, Trussell LO, Oertel D (1999) Time course and permeation of synaptic AMPA receptors in cochlear nuclear neurons correlate with input. J Neurosci 19:8721-8729.

Gauck V, Jaeger D (2000) The control of rate and timing of spikes in the deep cerebellar nuclei by inhibition. J Neurosci 20:3006-3016.

Goldberg JM, Brown PB (1969) Response of binaural neurons of dog superior olivary complex to dichotic tonal stimuli: some physiological mechanisms of sound localization. J Neurophysiol 32:613-636.

Hopkins WF, Allen ML, Houamed KM, Tempel BL (1994) Properties of voltage-gated $\mathrm{K}^{+}$currents expressed in Xenopus oocytes by mKv1.1, mKv1.2 and their heteromultimers as revealed by mutagenesis of the dendrotoxin-binding site in mKv1.1. Pflügers Arch 428:382-390.

Hunter JD, Milton JG, Thomas PJ, Cowan JD (1998) Resonance effect for neural spike time reliability. J Neurophysiol 80:1427-1438.

Jeffress LA (1948) A place theory of sound localization. J Comp Physiol Psychiatry 41:35-39.

Joris PX, Smith PH, Yin TC (1994) Enhancement of neural synchronization in the anteroventral cochlear nucleus. II. Responses in the tuning curve tail. J Neurophysiol 71:1037-1051.

Liberman MC (1978) Auditory-nerve response from cats raised in a low-noise chamber. J Acoust Soc Am 63:442-455.

Liberman MC (1982) Single-neuron labeling in the cat auditory nerve. Science 216:1239-1241.

Liberman MC (1991) Central projections of auditory-nerve fibers of differing spontaneous rate. I. Anteroventral cochlear nucleus. J Comp Neurol 313:240-258.

Liberman MC (1993) Central projections of auditory nerve fibers of differing spontaneous rate. II. Posteroventral and dorsal cochlear nuclei. J Comp Neurol 327:17-36.

Lytton WW, Sejnowski TJ (1991) Simulations of cortical pyramidal neurons synchronized by inhibitory interneurons. J Neurophysiol 66:1059-1079.
Mainen ZF, Sejnowski TJ (1995) Reliability of spike timing in neocortical neurons. Science 268:1503-1507.

Manis PB, Marx SO (1991) Outward currents in isolated ventral cochlear nucleus neurons. J Neurosci 11:2865-2880.

Manwani A, Koch C (1999) Detecting and estimating signals in noisy cable structures. II. Information theoretical analysis. Neural Comput 11:1831-1873.

Oertel D (1983) Synaptic responses and electrical properties of cells in brain slices of the mouse anteroventral cochlear nucleus. J Neurosci 3:2043-2053.

Oertel D, Bal R, Gardner SM, Smith PH, Joris PX (2000) Detection of synchrony in the activity of auditory nerve fibers by octopus cells of the mammalian cochlear nucleus. Proc Natl Acad Sci USA 97:11773-11779.

Press WH, Teukolsky SA, Vetterling WT, Flannery BP (1992) Numerical recipes in $\mathrm{C}$. The art of scientific computing, pp 827-888. Cambridge, UK: Cambridge UP.

Raman IM, Trussell LO (1992) The kinetics of the response to glutamate and kainate in neurons of the avian cochlear nucleus. Neuron 9:173-186.

Rathouz M, Trussell LO (1998) Characterization of outward currents in neurons of the avian nucleus magnocellularis. J Neurophysiol 80:2824-2835.

Reyes A, Rubel EW, Spain WJ (1994) Membrane properties underlying the firing of neurons in the avian cochlear nucleus. J Neurosci 14:5352-5364.

Reyes A, Rubel EW, Spain WJ (1996) In vitro analysis of optimal stimuli for phase-locking and time-delayed modulation of firing in avian nucleus laminaris neurons. J Neurosci 16:993-1007.

Robertson B, Owen D, Stow J, Butler C, Newland C (1996) Novel effects of dendrotoxin homologues on subtypes of mammalian Kv1 potassium channels expressed in Xenopus oocytes. FEBS Lett 383:26-30.

Rothman JS, Young ED (1996) Enhancement of neural synchronization in computational models of ventral cochlear nucleus bushy cells. Aud Neurosci 2:47-62.

Ryan AF, Miller JM, Pfingst BE, Martin GK (1984) Effects of reaction time performance on single-unit activity in the central auditory pathway of the rhesus macaque. J Neurosci 4:298-308.

Sada H, Ban T, Fujita T, Ebina Y, Sperelakis N (1995) Developmental change in fast $\mathrm{Na}$ channel properties in embryonic chick ventricular heart cells. Can J Physiol Pharmacol 73:1475-1484.

Schmid S, Guenther E (1998) Alterations in channel density and kinetic properties of the sodium current in retinal ganglion cells of the rat during in vivo differentiation. Neuroscience 85:249-258.

Sharp AA, O'Neil MB, Abbott LF, Marder E (1993) The dynamic clamp: artificial conductances in biological neurons. Trends Neurosci 16:389-394.

Smith PH (1995) Structural and functional differences distinguish principal from nonprincipal cells in the guinea pig MSO slice. J Neurophysiol 73:1653-1667.

Softky W (1994) Sub-millisecond coincidence detection in active dendritic trees. Neuroscience 58:13-41.

Softky W, Koch C (1993) The highly irregular firing of cortical cells is inconsistent with temporal integration of random EPSPs. J Neurosci $13: 334-350$.

Svirskis G, Kinzel J (2003) Influence of subthreshold nonlinearities on signal-to-noise ratio and timing precision for small signals in neurons. Minimal model analysis. Network, in press.

Trussell LO (1999) Synaptic mechanisms for coding timing in auditory neurons. Annu Rev Physiol 61:477-496.

Wang FC, Bell N, Reid P, Smith LA, McIntosh P, Robertson B, Dolly JO (1999) Identification of residues in dendrotoxin $\mathrm{K}$ responsible for its discrimination between neuronal $\mathrm{K}^{+}$channels containing Kv1.1 and 1.2 alpha subunits. Eur J Biochem 263:222-229.

Wiesenfeld K, Moss F (1995) Stochastic resonance and the benefits of noise: from ice ages to crayfish and SQUIDs. Nature 373:33-36.

Young SR, Rubel EW (1986) Embryogenesis of arborization pattern and topography of individual axons in N. laminaris of the chicken brain stem. J Comp Neurol 254:425-459.

Zhang X, Heinz MG, Bruce IC, Carney LH (2001) A phenomenological model for the responses of auditory-nerve fibers. I. Nonlinear tuning with compression and suppression. J Acoust Soc Am 109:648-669. 